\title{
Spin selection in single-frequency two-photon excitation of alkali-metal atoms
}

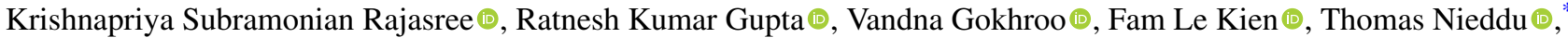 \\ Tridib Ray, ${ }^{*}$ Síle Nic Chormaic ${ }^{\circledR},{ }^{\dagger}$ and Georgiy Tkachenko $\bullet$ \\ Okinawa Institute of Science and Technology Graduate University, Onna, Okinawa 904-0495, Japan
}

(Received 15 April 2020; accepted 10 August 2020; published 31 August 2020)

\begin{abstract}
We develop a theoretical framework for spin selection in single-frequency two-photon excitation of alkalimetal atoms as a function of polarization of the excitation light. We verify the theory by experimentally probing the $5 S_{1 / 2} \rightarrow 6 S_{1 / 2}$ transition rate in ${ }^{87} \mathrm{Rb}$ in two configurations: paraxial light excitation of warm vapor and nonparaxial excitation of laser-cooled atoms. The transition rate follows a quadratic dependence on the helicity parameter linked to the excitation light's polarization. For paraxial excitation, the transition rate scales as the squared degree of linear polarization, being zero for circularly polarized light. In contrast, for nonparaxial excitation via an optical nanofiber, the two-photon transition is not completely extinguished by varying the light polarization. Our findings lead to a deeper and more universal understanding of the physics of multiphoton processes in atoms.
\end{abstract}

DOI: 10.1103/PhysRevResearch.2.033341

\section{INTRODUCTION}

Two-photon excitation, explored theoretically in 1931 [1] and first experimentally demonstrated in the early 1960s [2,3], revolutionized the fields of spectroscopy [4,5], fluorescence microscopy [6], and optical metrology [7]. Unlike singlephoton processes, two-photon excitation gives access to energy transitions which are electric dipole forbidden. In addition, if the simultaneously absorbed photons have the same frequency and are provided by counterpropagating beams, the fluorescence spectrum is free of Doppler broadening [8-11]; thus hyperfine transition lines can be clearly seen, allowing one to achieve a robust frequency reference $[12,13]$.

Two-photon transitions in atoms can only occur between electron orbitals of the same parity. This leads to a selection rule for the allowed change of the orbital angular momentum of the electron: $\Delta L=0, \pm 2$. In addition, the hyperfine energy levels involved in the transition obey a selection rule for the total angular momentum quantum number: $|\Delta F| \leqslant 2$ [14]. Here we focus on the spin selection rule, which further restricts the angular momentum changes in electric dipole allowed, single-frequency two-photon transitions between $S$ levels in atoms where the intermediate level is detuned from the single-photon resonance frequency. In this case, $\Delta F=0$ and $\Delta m_{F}=0$ (with $m_{F}$ being the magnetic quantum number) apply, meaning that the total spin of the atom must be preserved. If we assume that the spin of light is well defined,

\footnotetext{
* Present address: Laboratoire Kastler Brossel, Sorbonne Université, CNRS, ENS, Université PSL, Collège de France, 4 Place Jussieu, 75005 Paris, France.

†Corresponding author: sile.nicchormaic@ oist.jp

Published by the American Physical Society under the terms of the Creative Commons Attribution 4.0 International license. Further distribution of this work must maintain attribution to the author(s) and the published article's title, journal citation, and DOI.
}

conservation of angular momentum in the excitation process requires that the two photons must have mutually canceling spin projections along the quantization axis.

This principle has been verified experimentally using sodium [10,11] or rubidium [12,13] vapor illuminated by counterpropagating Gaussian beams. Doppler-free transition peaks were observed when the beams had equal linear polarizations or opposite circular polarizations in the laboratory frame, but the peaks disappeared for circular polarizations of the same handedness. When the excitation light is elliptically polarized, two-photon transitions are not completely absent, but occur at a rate that depends on the shape of the polarization ellipse, as demonstrated experimentally [13].

It is important to note that, in the aforementioned spectroscopy experiments, the atoms interacted with paraxial freespace electromagnetic fields. In recent years, there has been significant interest in shifting toward light fields confined at the micro- or even nanoscale $[15,16]$. In one of the more popular systems, neutral atoms are coupled to the evanescent field of a vacuum-clad optical nanofiber (ONF) [17-24]. Strong confinement of light around the ultrathin ONF waist region has led to demonstrations of two-photon and nonlinear atomic processes at ultralow excitation powers [25-28], including an electric quadrupole transition driven by a few microwatts [29]. Owing to the recent achievements of full polarization control for light guided in single-mode nanofibers [30-32], spin selection for two-photon excitation in ONF-coupled atoms can finally be explored experimentally.

In this work we first develop a theoretical model to describe the spin selection for two-photon transitions as a function of the polarization of the excitation light to go beyond the limiting cases of purely linear or purely circular polarization. As a verification tool, we experimentally study the polarization dependence of an $S \rightarrow S$ two-photon transition in a ${ }^{87} \mathrm{Rb}$ gas for two conceptually different excitation conditions: (i) warm atoms in a vapor cell with Gaussian beam illumination and (ii) laser-cooled atoms in the evanescent field of a single-mode ONF, where the light is strongly nonparaxial. 


\section{MODEL: TWO-PHOTON TRANSITION RATE}

Let us consider an atomic transition from a lower state $|g\rangle$ to an upper state $|e\rangle$ (with corresponding frequency $\omega_{e g}=$ $\left.\omega_{e}-\omega_{g}\right)$ excited by the simultaneous absorption of photons from two light fields characterized by a frequency $\omega_{j=1,2}$, an amplitude $\mathcal{E}_{j}$, and a unit polarization vector $\mathbf{u}_{j}$. The rate of such a two-photon transition is given by [33]

$$
\begin{aligned}
P_{g e}= & C \mid \frac{1}{\hbar} \sum_{i}\left(\frac{\left\langle e\left|\mathbf{u}_{1} \cdot \mathbf{d}\right| i\right\rangle\left\langle i\left|\mathbf{u}_{2} \cdot \mathbf{d}\right| g\right\rangle}{\omega_{i g}-\omega_{2}+i \Gamma_{i} / 2}\right. \\
& \left.+\frac{\left\langle e\left|\mathbf{u}_{2} \cdot \mathbf{d}\right| i\right\rangle\left\langle i\left|\mathbf{u}_{1} \cdot \mathbf{d}\right| g\right\rangle}{\omega_{i g}-\omega_{1}+i \Gamma_{i} / 2}\right)\left.\right|^{2},
\end{aligned}
$$

with

$$
C=\frac{1}{16 \hbar^{2}} \frac{\Gamma}{\left(\omega_{1}+\omega_{2}-\omega_{e g}\right)^{2}+(\Gamma / 2)^{2}}\left|\mathcal{E}_{1}\right|^{2}\left|\mathcal{E}_{2}\right|^{2},
$$

where $\mathbf{d}$ is the atomic dipole operator, $|i\rangle$ is an intermediate state, $\omega_{i g}=\omega_{i}-\omega_{g}$ is the frequency of the atomic transition between the states $|i\rangle$ and $|g\rangle, \Gamma=\Gamma_{e}+\Gamma_{g}$ is the sum of the decay rates of the upper and lower states, and $\Gamma_{e}, \Gamma_{g}$, and $\Gamma_{i}$ are the natural linewidths of the states $|e\rangle,|g\rangle$, and $|i\rangle$, respectively. We find

$$
P_{g e}=C\left|V_{e g}\right|^{2}
$$

where

$$
V_{e g}=\sum_{K=0,1,2} V_{e g}^{(K)}
$$

is the matrix element of the two-photon transition operator $V[34,35]$, with the scalar $(K=0)$, vector $(K=1)$, and tensor $(K=2)$ parts given as

$$
\begin{aligned}
V_{e g}^{(K)}= & (-1)^{K+I+J_{e}-M_{g}} \sqrt{\left(2 F_{e}+1\right)\left(2 F_{g}+1\right)} \\
& \times\left\{\begin{array}{ccc}
F_{e} & K & F_{g} \\
J_{g} & I & J_{e}
\end{array}\right\} \alpha_{J}^{(K)} \\
& \times \sum_{q}\left\{\mathbf{u}_{1} \otimes \mathbf{u}_{2}\right\}_{K q}\left(\begin{array}{ccc}
F_{e} & K & F_{g} \\
M_{e} & q & -M_{g}
\end{array}\right),
\end{aligned}
$$

where $J$ is the electronic angular momentum quantum number, $M$ - electronic magnetic quantum number, $I$ - nuclear spin quantum number, and we have introduced the notation

$$
\begin{aligned}
\alpha_{J}^{(K)}= & (-1)^{K+J_{e}+J_{g}} \sqrt{2 K+1} \sum_{n_{i} J_{i}}\left\{\begin{array}{ccc}
1 & K & 1 \\
J_{e} & J_{i} & J_{g}
\end{array}\right\} \\
& \times\left\langle n_{e} J_{e}\|\mathbf{d}\| n_{i} J_{i}\right\rangle\left\langle n_{i} J_{i}\|\mathbf{d}\| n_{g} J_{g}\right\rangle \\
& \times\left[\frac{1}{\hbar\left(\omega_{n_{i} J_{i}}-\omega_{g}-\omega_{2}+i \Gamma_{i} / 2\right)}\right. \\
& \left.+\frac{(-1)^{K}}{\hbar\left(\omega_{n_{i} J_{i}}-\omega_{g}-\omega_{1}+i \Gamma_{i} / 2\right)}\right] .
\end{aligned}
$$

Here $\alpha_{J}^{(K)}$ are the reduced scalar $(K=0)$, vector $(K=1)$, and tensor $(K=2)$ two-photon susceptibility coefficients in the basis $J$. It is clear from Eq. (6) that, in the particular case of equal photon frequencies $\omega_{1}=\omega_{2}$, we have $\alpha_{J}^{(1)}=0$. In this case, the vector part $V^{(1)}$ of the two-photon transition operator $V$ vanishes.

The general selection rule for the electronic orbital angular momentum quantum number $L$ is that the two-photon transition can be allowed only if $\left|L_{e}-L_{g}\right|=0,2$. It follows from this condition that the two-photon transition can be allowed only if the initial state $|g\rangle$ and the final state $|e\rangle$ have the same parity, opposite to that of the intermediate states $|i\rangle$. This parity rule is a consequence of the odd character of the electric dipolar coupling. The parity rule for two-photon excitation is the opposite of the rule for single-photon excitation.

The general selection rule for the electronic angular momentum quantum number $J$ is that the two-photon transition can be allowed only if $\left|J_{e}-J_{g}\right| \leqslant 2$ [36-38]. In the case of equal photon frequencies, the transitions $J_{g}=0 \rightarrow J_{e}=1$ and $J_{g}=1 \rightarrow J_{e}=0$ are forbidden [36].

In the case where $J_{e} \neq J_{g}$, the Wigner 6- $j$ symbol in Eq. (6) for $K=0$ is zero. This means that the coefficient $\alpha_{J}^{(0)}$ and the corresponding matrix element $V_{e g}^{(0)}$ of the scalar part of the two-photon transition operator $V$ vanish for $J_{e} \neq J_{g}$.

In the case where $J_{e}=J_{g}=0$ or $1 / 2$, the Wigner $6-j$ symbol in Eq. (6) for $K=2$ is zero. This means that the coefficient $\alpha_{J}^{(2)}$ and the corresponding matrix element $V_{e g}^{(2)}$ of the tensor part of the two-photon transition operator $V$ vanish for $J_{e}=J_{g}=0$ or $1 / 2$.

The aforementioned selection rules for the electronic angular momentum $J$ are equally true for the total angular momentum $F$. However, depending on $J$, we may find additional selection rules for $F$. For instance, when $J_{e}=J_{g}=0$ or $1 / 2$ and the photons have equal frequencies, we have the selection rules $F_{e}=F_{g}$ and $M_{e}=M_{g}$ [36].

The average transition rate for the transition between the hyperfine levels $F_{g}$ and $F_{e}$ is given by

$$
P_{F_{g} F_{e}}=\frac{1}{2 F_{g}+1} \sum_{M_{g} M_{e}} P_{g e} .
$$

In the absence of a magnetic field, the level energies $\hbar \omega_{g}$ and $\hbar \omega_{e}$ do not depend on $M_{g}$ and $M_{e}$, respectively. In this case, we find

$$
\begin{aligned}
P_{F_{g} F_{e}}= & C\left(2 F_{e}+1\right) \sum_{K}\left\{\begin{array}{ccc}
F_{e} & K & F_{g} \\
J_{g} & I & J_{e}
\end{array}\right\}^{2} \\
& \times \frac{\left|\alpha_{J}^{(K)}\right|^{2}}{2 K+1} \sum_{q}\left|\left\{\mathbf{u}_{1} \otimes \mathbf{u}_{2}\right\}_{K q}\right|^{2} .
\end{aligned}
$$

If $J_{e}=J_{g}=0$ or $1 / 2$ and $\omega_{1}=\omega_{2}$, the two-photon operator is scalar. In this case, the transition may occur only for $F_{e}=F_{g}$ and hence we find

$$
P_{F_{g} F_{e}}=\frac{C}{3\left(2 J_{g}+1\right)}\left|\alpha_{J}^{(0)}\right|^{2}\left|\left(\mathbf{u}_{1} \cdot \mathbf{u}_{2}\right)\right|^{2} .
$$

It is interesting to note that $P_{F_{g} F_{e}}$ does not depend on $F_{g}$. The line intensity $I_{F_{g} F_{e}}$ is the product of the transition rate $P_{F_{g} F_{e}}$ and the number of atoms in the initial state $\left|n_{g} J_{g} F_{g}\right\rangle$. If the hyperfine sublevels of the ground state are populated according to their degeneracy, the line intensity is $I_{F_{g} F_{e}}=P_{F_{g} F_{e}} N_{0}\left(2 F_{g}+\right.$ $1) /\left(2 J_{g}+1\right)(2 I+1)$, where $N_{0}$ is the total number of atoms 
in the ground state. It is clear that $I_{F_{g} F_{e}}$ is proportional to $2 F_{g}+1$ [36].

According to Eq. (9), the dependence of the two-photon transition rate $P_{F_{g} F_{e}}$ on the polarization of the excitation light field is determined by the factor $\left|\left(\mathbf{u}_{1} \cdot \mathbf{u}_{2}\right)\right|^{2}$. The maximal value of this factor is 1 , achieved for two fields with identical linear polarizations or opposite circular polarizations with respect to the quantization axis $z$ (that is, opposite photon spin projections onto $z$ ). The minimal value of $\left|\left(\mathbf{u}_{1} \cdot \mathbf{u}_{2}\right)\right|^{2}$ is 0 and is achieved for two fields with orthogonal linear polarizations or identical circular polarizations with respect to $z$ (identical photon spin projections onto $z$ ).

In practice, we only consider the two-photon transition with $J_{e}=J_{g}=1 / 2$, under excitation by two fields with identical frequencies $\left(\omega_{1}=\omega_{2}=\omega_{e g} / 2\right)$, amplitudes $\left(\mathcal{E}_{1}=\mathcal{E}_{2}=\right.$ $\mathcal{E} / \sqrt{2}$ ), and polarizations $\left(\mathbf{u}_{1}=\mathbf{u}_{2}=\mathbf{u}\right)$. The fields are either copropagating (which we refer to as a one-beam configuration) or counterpropagating (two-beam configuration). For such a single-frequency two-photon excitation, the transition rate is

$$
P_{g e}=\frac{1}{96 \hbar^{2} \Gamma}\left|\alpha_{J}^{(0)}\right|^{2} \xi,
$$

where $\xi=|(\mathcal{E} \cdot \mathcal{E})|^{2}=|\mathcal{E}|^{4}|(\mathbf{u} \cdot \mathbf{u})|^{2}$ expresses both of the characteristic features of the two-photon process: the quadratic dependence on the field intensity $|\mathcal{E}|^{2}$ and the polarization dependence, which is the main object in this study.

Now let us consider excitation of this two-photon transition in a cool gas of atoms in the evanescent field of an optical nanofiber, in particular by an elliptically polarized fundamental mode propagating in the $+z$ direction of the Cartesian coordinate system $(x, y, z)$. The electric part of the field in this mode is given by

$$
\mathcal{E}=\left(\sqrt{1+\sigma} \mathcal{E}_{+1}+\sqrt{1-\sigma} \mathcal{E}_{-1}\right) / \sqrt{2},
$$

where $\sigma \in[-1,1]$ is the helicity parameter $[39,40]$ and

$$
\mathcal{E}_{p}=\left(e_{r} \hat{r}+p e_{\varphi} \hat{\varphi}+e_{z} \hat{z}\right) e^{i(p \varphi+\beta z)},
$$

where $p= \pm 1$ is the polarization index for quasicircularly polarized fundamental guided modes [41], $\beta$ is the propagation constant, and $e_{r}, e_{\varphi}$, and $e_{z}$ are the reduced cylindrical components of the mode function and they are independent of $\varphi$ and $z$ [42-44]. Note that if the field is retroreflected, one must change the sign for $\beta, \sigma$, and $p$. Given Eqs. (12) and (11), the scalar product

$$
\begin{aligned}
(\mathcal{E} \cdot \mathcal{E})= & \left(\cos 2 \varphi+i \sigma \sin 2 \varphi+\sqrt{1-\sigma^{2}}\right)\left(e_{r}^{2}+e_{z}^{2}\right) e^{2 i \beta z} \\
& +\left(\cos 2 \varphi+i \sigma \sin 2 \varphi-\sqrt{1-\sigma^{2}}\right) e_{\varphi}^{2} e^{2 i \beta z}
\end{aligned}
$$

The mode function components have the properties $e_{r}^{2}=$ $-\left|e_{r}\right|^{2}, e_{\varphi}^{2}=\left|e_{\varphi}\right|^{2}$, and $e_{z}^{2}=\left|e_{z}\right|^{2}[42-45]$. Hence, we find

$$
\begin{aligned}
\xi= & {\left[\sqrt{1-\sigma^{2}}\left(\left|e_{z}\right|^{2}-\left|e_{r}\right|^{2}-\left|e_{\varphi}\right|^{2}\right)\right.} \\
& \left.+\left(\left|e_{z}\right|^{2}-\left|e_{r}\right|^{2}+\left|e_{\varphi}\right|^{2}\right) \cos 2 \varphi\right]^{2} \\
& +\sigma^{2}\left(\left|e_{z}\right|^{2}-\left|e_{r}\right|^{2}+\left|e_{\varphi}\right|^{2}\right)^{2} \sin ^{2} 2 \varphi .
\end{aligned}
$$

We neglect the interaction and collisions between the atoms and assume that the gas volume is a cylindrical shell with $z \in[0, \mathcal{L}], \varphi \in[0,2 \pi]$, and $r \in\left[a, r_{\max }\right]$. Here $a$ is the fiber radius and $\mathcal{L}$ and $r_{\max }$ are, respectively, the length and radius of the atomic cloud. We assume that the atoms have the same flat stochastic distribution of position. To estimate the transition rate, we must average the factor $\xi$ over the position of atoms.

Since $\xi$ does not depend on the axial coordinate $z$, statistical averaging with respect to $z$ does not affect $\xi$. Statistical averaging with respect to the azimuthal angle $\varphi$ can be carried out by using the formulas $\left\langle\cos ^{2} 2 \varphi\right\rangle_{\varphi}=\left\langle\sin ^{2} 2 \varphi\right\rangle_{\varphi}=1 / 2$ and $\langle\cos 2 \varphi\rangle_{\varphi}=\langle\sin 2 \varphi\rangle_{\varphi}=0$. Here we have introduced the notation $\langle\cdots\rangle_{\varphi}=(2 \pi)^{-1} \int_{0}^{2 \pi} \cdots d \varphi$ for statistical averaging with respect to $\varphi$. Hence, we find

$$
P_{g e} \propto \bar{\xi}=A-\sigma^{2} B,
$$

where

$$
\begin{aligned}
A= & \left\langle\left(\left|e_{r}\right|^{2}+\left|e_{\varphi}\right|^{2}-\left|e_{z}\right|^{2}\right)^{2}\right\rangle_{r} \\
& +0.5\left\langle\left(\left|e_{r}\right|^{2}-\left|e_{\varphi}\right|^{2}-\left|e_{z}\right|^{2}\right)^{2}\right\rangle_{r}, \\
B= & \left\langle\left(\left|e_{r}\right|^{2}+\left|e_{\varphi}\right|^{2}-\left|e_{z}\right|^{2}\right)^{2}\right\rangle_{r} \\
& -0.5\left\langle\left(\left|e_{r}\right|^{2}-\left|e_{\varphi}\right|^{2}-\left|e_{z}\right|^{2}\right)^{2}\right\rangle_{r},
\end{aligned}
$$

with $\langle\cdots\rangle_{r}$ denoting statistical averaging over the radial distance $r$ from the nanofiber. The quadratic term in Eq. (15) predicts that the transition rate is maximum for linearly polarized $(\sigma=0)$ and minimum (but, in general, nonzero) for circularly polarized $(\sigma= \pm 1)$ excitation.

\section{EXPERIMENT: WARM ATOMS EXCITED BY PARAXIAL LIGHT}

In order to verify the above theoretical result experimentally, we choose the $5 S_{1 / 2}(F=2) \rightarrow 6 S_{1 / 2}\left(F^{\prime}=2\right)$ transition in ${ }^{87} \mathrm{Rb}$ [see Fig. 1(a)], accessible via two-photon excitation at $993 \mathrm{~nm}$ [12]. The excitation light is provided by a Ti:sapphire laser and its frequency is fine-tuned via spectroscopy in a vapor cell containing a natural mixture of ${ }^{85} \mathrm{Rb}$ and ${ }^{87} \mathrm{Rb}$ atoms, maintained at $100^{\circ} \mathrm{C}$. A schematic of the experiment is given in Fig. 1(b). The polarization of the excitation beam is given by a unit vector $\mathbf{s}=\left(1, S_{1}, S_{2}, S_{3}\right)$, where $S_{1,2,3}$ are the reduced Stokes parameters defined from the point of view of the receiver. A $993-\mathrm{nm}$ laser beam of $150 \mathrm{~mW}$ is weakly focused into the vapor cell by a convex lens L1 (focal distance $f_{1}=150 \mathrm{~mm}$ ) and the 780-nm and the 795-nm fluorescence (from the $5 P_{1 / 2} \rightarrow 5 S_{1 / 2}$ and the $5 P_{3 / 2} \rightarrow 5 S_{1 / 2}$ decay paths, respectively) emitted around the focal point is detected by means of a photomultiplier tube (PMT) through a relay telescope and a shortpass filter (Thorlabs model No. FES0800, not shown). The fluorescence intensity is a measure of the two-photon transition rate. Figure 1(c) (top panel) depicts the spectroscopy signal collected for horizontal polarization, $\mathbf{H}=(1,1,0,0)$ (note that a low-pass digital frequency filter was applied to the data in order to suppress the noise). To convert this one-beam configuration into the two-beam case, we add a concave mirror $\left(f_{\mathrm{CM}}=f_{1} / 2=75 \mathrm{~mm}\right)$ placed at $2 f_{1}$ away from L1. As a result, the spectrum reveals the 200-fold increased Doppler-free peaks [see the bottom panel in Fig. 1(c), where each hyperfine transition of ${ }^{85} \mathrm{Rb}$ and ${ }^{87} \mathrm{Rb}$ is indicated with the corresponding $F \leftrightarrow F^{\prime}$ values]. 


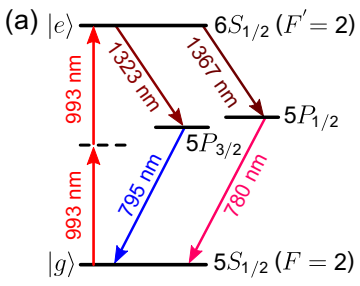

(c)

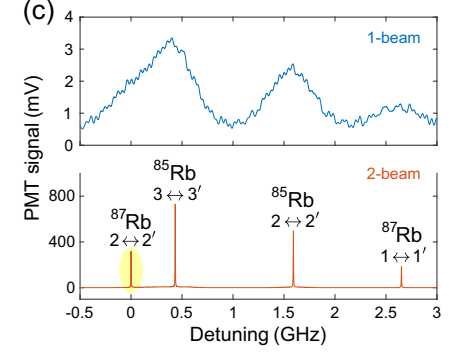

(b)

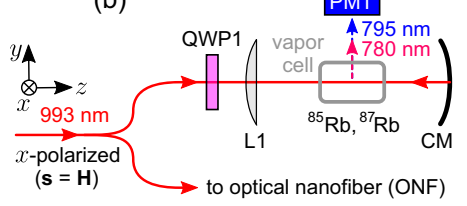

(d)

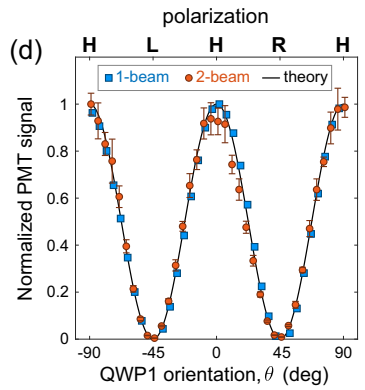

FIG. 1. (a) Simplified energy level diagram for ${ }^{87} \mathrm{Rb}$ as relevant to the experiment. (b) Vapor cell spectroscopy setup: QWP1, quarter waveplate; L1, convex lens; CM, concave mirror; and PMT, photomultiplier tube. (c) Spectroscopy signal (background subtracted) measured in the one-beam (top) and two-beam (bottom) configurations. Labeled peaks correspond to the Doppler-free hyperfine transitions, with the chosen one $\left(2 \leftrightarrow 2^{\prime}\right.$ in $\left.{ }^{87} \mathrm{Rb}\right)$ highlighted. (d) Polarization dependence of the fluorescence signal from the chosen transition. Squares and circles show the experimental data for onebeam and two-beam configurations, respectively. The solid line shows the results of simulation. The principal polarization states of the 993-nm light are indicated: horizontal (H), left-handed circular (L), and right-handed circular $(\mathbf{R})$.

Fluorescence signals from the vapor cell for both the onebeam and two-beam configurations should follow Eq. (15), irrespective of the Doppler broadening in the former case. To test this, we introduce a quarter waveplate QWP1 before L1 and scan the helicity parameter $\sigma$ over the whole $[-1,1]$ range by varying $\theta$, the angle between $x$ and the slow axis of the waveplate. As a result, the initial horizontal polarization state is transformed into $\left(1, \cos ^{2} 2 \theta, \sin 2 \theta \cos 2 \theta,-\sin 2 \theta\right)$ and $\sigma=-S_{3}=\sin 2 \theta$. Since the Gaussian field is paraxial, we assume $e_{z}=0$ and $\left|e_{r}\right|=\left|e_{\varphi}\right|$. Thence, Eq. (16) gives $A=B$ and Eq. (15) reduces to $P_{g e} \propto 1-\sigma^{2}$. The two-photon transition rate is expected to scale as $\cos ^{2} 2 \theta$, reaching zero at $\theta=(2 n+1) \pi / 4$ with $n \in \mathbb{Z}$, as confirmed by the measured polarization dependence of the fluorescence for the chosen two-photon transition [see Fig. 1(d)]. In the one-beam case (blue squares), the signal is defined simply as the mean voltage output of the PMT and the confidence range is smaller than the marker size. In the two-beam configuration, the transition spectral profile is fitted to a Lorentzian curve [46] and the markers (orange circles) shown in Fig. 1(d) are the average maxima of the fitted curves, while the error bars indicate the standard deviation range over ten independent measurements. For convenience of presentation, each data set in Fig. 1(d) is normalized to the maximum.

It is important to note that the results obtained with the hot vapor cell can be explained in simpler terms. Since the transition is allowed only when the excitation light carries zero net spin angular momentum, $P_{g e}$ is intuitively expected to be linked to the degree of linear polarization (DOLP) $\mathcal{D}=$

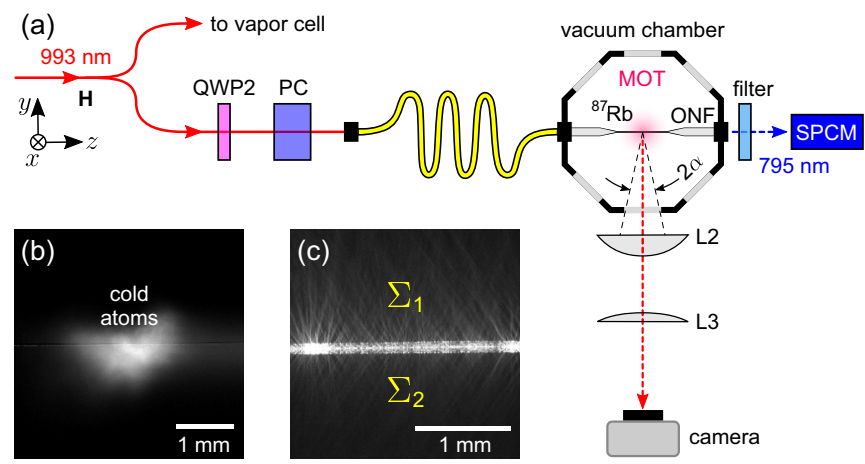

FIG. 2. (a) Atom-nanofiber experimental setup: QWP2, quarter waveplate; PC, polarization compensator; MOT, magneto-optical trap; L2 and L3, convex lenses; and SPCM, single-photon counting module. (b) Fluorescence image of the cold-atom cloud around the nanofiber. (c) Excitation light scattered at the nanofiber waist. Here $\Sigma_{1}$ and $\Sigma_{2}$ are the brightness sums of the upper and lower halves of the image.

$\sqrt{S_{1}^{2}+S_{2}^{2}}$. Physically, the DOLP is the maximum fraction of optical power one can measure in transmission through a lossless linear polarizer. Owing to the quadratic power dependence of the two-photon transition rate, $P_{g e} \propto \mathcal{D}^{2}=$ $\cos ^{4} 2 \theta+\sin ^{2} 2 \theta \cos ^{2} 2 \theta=\cos ^{2} 2 \theta$, which we indeed confirm experimentally. Continuing this line of thought, we notice that the mentioned lossless linear polarizer defines the DOLP for both the forward-propagating beam and the retroreflected one. Thus, if the beams are linearly polarized in planes tilted by an angle $\delta$ with respect to each other, the two-photon transition rate would scale as $\mathcal{D}^{2}=\cos ^{2} \delta$, which is equivalent to Malus's law. This result explains the absence of Doppler-free peaks in the crossed polarization $\left(\pi-\pi^{\prime}\right)$ case experimentally tested in [12], where the setup contained a quarter waveplate between the vapor cell and the concave mirror.

\section{EXPERIMENT: LASER-COOLED ATOMS EXCITED BY A NANOFIBER MODE}

Now let us consider the case where excitation is by the evanescent field of light guided in an optical nanofiber. The experimental setup is sketched in Fig. 2(a). The ONF is fabricated from a commercial single-mode optical fiber (Fibercore model No. SM800-5.6-125) via a flame-brushing technique [47]. The initial fiber diameter of $125 \mu \mathrm{m}$ is exponentially tapered to $400 \mathrm{~nm}$ at the waist, thereby supporting only the fundamental guided modes for both the 993-nm and 795-nm wavelengths (the transmission at 993 $\mathrm{nm}$ is about $30 \%$ including the coupling losses). The ONF is mounted in an ultrahigh vacuum chamber and a standard three-beam retroreflected magneto-optical trap (MOT) is used to produce the cold ${ }^{87} \mathrm{Rb}$ atoms at the nanofiber waist (see [48] for details). An average atom cloud density of $10^{9} \mathrm{~cm}^{-3}$ is estimated from fluorescence imaging of the cold atoms [see Fig. 2(b)], using a CCD camera (Andor Luca ${ }^{\mathrm{EM}} \mathrm{R}$ model No. DL-604M-OEM) and a microscope composed of two convex lenses $\mathrm{L} 2\left(f_{2}=125 \mathrm{~mm}\right)$ and $\mathrm{L} 3\left(f_{3}=250 \mathrm{~mm}\right)$. The 993-nm light is sent through the fiber for $2 \mathrm{~h}$ prior to measurements in order to heat the ONF and thus prevent 
(a)

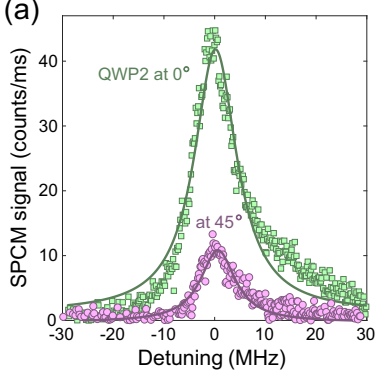

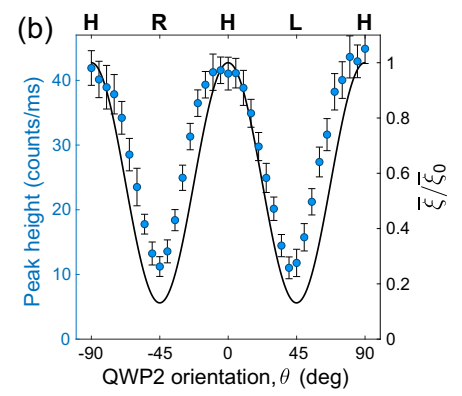

FIG. 3. Measured 795-nm fluorescence from the cold atoms. (a) Typical transition peaks for a linearly polarized (QWP2 at $\theta=0^{\circ}$ and $\sigma=0)$ and a circularly polarized $\left(\theta=45^{\circ}\right.$ and $\left.\sigma=1\right)$ input 993-nm beam. The solid lines represent Lorentzian curve fitting. (b) Polarization dependence of the 795-nm fluorescence. The solid line shows the results of simulation.

deposition of atoms onto its surface. The optical power, measured at the output end of the fiber, is maintained at 0.6 $\mathrm{mW}$ during the experiment. In a typical experiment sequence, the MOT is loaded to saturation and then the excitation laser is scanned around the chosen transition, while the 795-nm fluorescence is coupled into the nanofiber and recorded by a single-photon counting module (SPCM) (Excelitas Technologies model No. SPCM-AQRH-14-FC) through a bandpass filter. In a similar manner to the vapor cell tests, in this setup the input polarization is varied by means of a quarter waveplate QWP2 placed before the fiber coupler. In a steady state (i.e., after the initial 2-h heating stage and when QWP2 is not rotating), the polarization is stable within $1^{\circ}$ on the Poincaré sphere, as confirmed by a free-space polarimeter (Thorlabs model No. PAX1000IR) placed at the output pigtail of the fiber.

Due to stress-induced birefringence in the tapered fiber, the preset input polarization state of the 993-nm fiber-guided light is transformed into an unknown state at the nanofiber waist. In order to gain control over the helicity at the waist, we reverse the transformation using a polarization compensator introduced between QWP2 and the input pigtail of the fiber. The compensator consists of a variable retarder (liquid crystal type, slow axis parallel to $x$ ) and two quarter waveplates [30]. The compensation procedure is based on imaging the 993-nm light (through an $x$-oriented linear polarizer, not shown) scattered from natural imperfections of the nanofiber [32]. This method relies on identifying two nonorthogonal linear polarization states at the nanofiber waist, the first being horizontal (or vertical) and the second being diagonal (or antidiagonal). The latter corresponds to the absolute maximum (or minimum) of the intensity difference between two regions of the scattered light image, $\Delta=\Sigma_{1}-\Sigma_{2}$ [see Fig. 2(c)]. To maximize the precision of $\Delta$ measurements, we replace L2 by a convex lens with a diameter of $50 \mathrm{~mm}$ and a focal distance of $75 \mathrm{~mm}$, thus achieving a maximum collection angle $\alpha \approx 18^{\circ}$. The errors in the state identification are expected to be less than $10^{\circ}$ on the Poincaré sphere [32]. When $|\sigma|$ approaches unity (the most interesting case in this study), this error corresponds to a confidence range of about $1.5 \%$ for $|\sigma|$ and $3 \%$ for $\sigma^{2}$.

The experimental results are shown in Fig. 3, with Fig. 3(a) depicting the 795-nm fluorescence collected by the SPCM in the two limiting cases: the excitation light is (i) linearly polarized $\left(\sigma=0\right.$ and $\left.\bar{\xi}_{0}=A\right)$ and (ii) circularly polarized ( $\sigma= \pm 1$ and $\bar{\xi}_{ \pm 1}=A-B$ ). Each set of data is fitted to a Lorentzian curve [solid lines in Fig. 3(a)] and its peak value is the measure of the two-photon transition rate. Since guided modes of a nanofiber feature a nonvanishing longitudinal field component, $A \neq B$ and $\bar{\xi} \neq 0$, even when the input light is circularly polarized. The fluorescence measurements over the complete range of $\sigma$ are summarized in Fig. 3(b), where the solid curve is the calculated $\bar{\xi} / \bar{\xi}_{0}$ and the error bars indicate the standard deviation ranges obtained from 40 experimental sequences for every orientation of QWP2. The susceptibility coefficient was calculated as detailed in the Appendix.

\section{DISCUSSION}

We attribute the discrepancy between the experimental data and the theoretical curve, specifically the shallower and narrower dips in the measurement, to several experimental factors beyond our control: The polarization compensation only works for the transverse field components, atoms are not necessarily evenly distributed around the nanofiber [as suggested by the cloud picture in Fig. 2(b)], individual atoms may see local inhomogeneities of the excitation field near the ONF waist, or residual magnetic field and cooling beams in the excitation region may be influencing the two-photon process. The lateral shift of the rising slopes seen in both periods of the $\theta$ dependence in Fig. 3(b) is likely to be an experimental artifact such as imperfection of the waveplate, enhanced by coupling of light into the fiber. Other effects not taken into account are possible polarization-dependent saturation [49] of the transition in the atomic cloud, a polarization-induced inhomogeneity in the intensity profile [42] and the related change in the local atomic density due to the dipole force, and position-dependent Stark shifts in the atomic energy levels [50]. We also note that the relation $\sigma=\sin 2 \theta$ may not be exactly fulfilled for ONF-mediated excitation. For instance, the generation of orbital angular momentum in the evanescent field, which is more significant for quasicircular polarization [40,51], effectively changes the helicity and its relation to the polarization of light sent into the fiber. This invites further studies on two-photon processes under nonparaxial fields, inclusive of the orbital degree of freedom.

\section{CONCLUSION}

In this work we observed the spin selection rule as applied to an $S \rightarrow S$ two-photon atomic transition, both within and beyond the paraxial limit for the excitation light. In the latter case, the light was delivered by the evanescent field of a single-mode optical nanofiber, which also served as a detection channel for the fluorescence signal, a measure of the two-photon transition rate. Owing to the accurate polarization control at the nanofiber waist, we were able to study the transition rate as a function of helicity of the excitation. In contrast to the paraxial case, the two-photon transition in the evanescent field could not be extinguished by simply varying the polarization of the coupled light; we observed a minimum rate of about $13 \%$ of the maximum in theory and $25 \%$ in practice. These findings are expected to have impact 
in atom-based hybrid quantum technologies where full control on quantum state selection is vital and could open new ways of selecting transition pathways for frequency references and atomic clocks, novel fiber-based atom trapping schemes [52], transfer of spin or orbital angular momentum between light and atoms [53,54], chiral nanophotonics [24], and fundamental precision tests in parity nonconservation $[55,56]$.

\section{ACKNOWLEDGMENTS}

The authors thank Antoine Pichené and René Henke for assembling the hot vapor cell setup. This work was financially supported by the Okinawa Institute of Science and Technology Graduate University and the Japan Society for the Promotion of Science (JSPS) Grant-in-Aid for Scientific Research (C) Grant No. 19K05316. G.T. was supported by a JSPS International Research Fellowship (Standard, Grant No. P18367).

\section{APPENDIX: CALCULATION OF $\alpha_{J}^{(0)}$}

In our experiment, the two-photon transition occurs between the ground state $|g\rangle=\left|5 S_{1 / 2}\right\rangle$ and the excited state $|e\rangle=\left|6 S_{1 / 2}\right\rangle$ of Rb with $\omega_{1}=\omega_{2}=\omega=\omega_{e g} / 2$. We keep the contribution of the two most important intermediate states $\left|i_{1}\right\rangle=\left|5 P_{1 / 2}\right\rangle$ and $\left|i_{2}\right\rangle=\left|5 P_{3 / 2}\right\rangle$. Since the detunings are large, we neglect $\Gamma_{i}$. Then Eq. (6) yields

$\alpha_{J}^{(0)}=\frac{\sqrt{6}}{3 \hbar}\left(\frac{1}{\Delta_{1}}\left\langle e\|\mathbf{d}\| i_{1}\right\rangle\left\langle i_{1}\|\mathbf{d}\| g\right\rangle-\frac{1}{\Delta_{2}}\left\langle e\|\mathbf{d}\| i_{2}\right\rangle\left\langle i_{2}\|\mathbf{d}\| g\right\rangle\right)$,

where $\Delta_{1}=\omega-\left(\omega_{i_{1}}-\omega_{g}\right)$ and $\Delta_{2}=\omega-\left(\omega_{i_{2}}-\omega_{g}\right)$.

With the help of the relation $[34,35]$

$$
\begin{aligned}
\left\langle n L J\|\mathbf{d}\| n^{\prime} L^{\prime} J^{\prime}\right\rangle \equiv & \left\langle n L S J\|\mathbf{d}\| n^{\prime} L^{\prime} S J^{\prime}\right\rangle \\
= & (-1)^{S+L+J^{\prime}+1} \sqrt{(2 J+1)\left(2 J^{\prime}+1\right)} \\
& \times\left\{\begin{array}{ccc}
J & 1 & J^{\prime} \\
L^{\prime} & S & L
\end{array}\right\}\left\langle n L\|\mathbf{d}\| n^{\prime} L^{\prime}\right\rangle,
\end{aligned}
$$

we find

$$
\begin{aligned}
& \left\langle e\|\mathbf{d}\| i_{1}\right\rangle=\frac{\sqrt{6}}{3}\langle 6 S\|\mathbf{d}\| 5 P\rangle, \\
& \left\langle e\|\mathbf{d}\| i_{2}\right\rangle=\frac{2 \sqrt{3}}{3}\langle 6 S\|\mathbf{d}\| 5 P\rangle
\end{aligned}
$$

and

$$
\begin{aligned}
& \left\langle i_{1}\|\mathbf{d}\| g\right\rangle=-\frac{\sqrt{6}}{3}\langle 5 P\|\mathbf{d}\| 5 S\rangle, \\
& \left\langle i_{2}\|\mathbf{d}\| g\right\rangle=\frac{2 \sqrt{3}}{3}\langle 5 P\|\mathbf{d}\| 5 S\rangle .
\end{aligned}
$$

Inserting Eqs. (A3) and (A4) into Eq. (A2) yields

$$
\alpha_{J}^{(0)}=-\frac{2 \sqrt{6}}{9 \hbar}\left(\frac{1}{\Delta_{1}}+\frac{2}{\Delta_{2}}\right)\langle 6 S\|\mathbf{d}\| 5 P\rangle\langle 5 P\|\mathbf{d}\| 5 S\rangle .
$$

We note that $\left\langle e\|\mathbf{d}\| i_{1}\right\rangle$ and $\left\langle e\|\mathbf{d}\| i_{2}\right\rangle$ have the same sign, but $\left\langle i_{1}\|\mathbf{d}\| g\right\rangle$ and $\left\langle i_{2}\|\mathbf{d}\| g\right\rangle$ have opposite signs. Consequently, Eq. (A1) yields

$$
\begin{aligned}
\left|\alpha_{J}^{(0)}\right|= & \frac{\sqrt{6}}{3 \hbar}\left|\frac{1}{\Delta_{1}}\right|\left\langle e\|\mathbf{d}\| i_{1}\right\rangle\left\langle i_{1}\|\mathbf{d}\| g\right\rangle \mid \\
& +\frac{1}{\Delta_{2}}\left|\left\langle e\|\mathbf{d}\| i_{2}\right\rangle\left\langle i_{2}\|\mathbf{d}\| g\right\rangle\right| .
\end{aligned}
$$

Since $\left|\left\langle i_{1}\|\mathbf{d}\| g\right\rangle\right|=4.253$ a.u., $\left|\left\langle i_{1}\|\mathbf{d}\| e\right\rangle\right|=4.145$ a.u., $\left|\left\langle i_{2}\|\mathbf{d}\| g\right\rangle\right|=6.003$ a.u., and $\left|\left\langle i_{2}\|\mathbf{d}\| e\right\rangle\right|=6.047$ a.u. [57], we find the estimates $|\langle 5 P\|\mathbf{d}\| 5 S\rangle|=5.202$ a.u. and $|\langle 5 P\|\mathbf{d}\| 6 S\rangle|=5.184$ a.u. The lifetime of the state $6 S_{1 / 2}$ is $45.4 \mathrm{~ns}$. The lifetimes of the states $5 P_{1 / 2}$ and $5 P_{3 / 2}$ are 27.4 and $26 \mathrm{~ns}$, respectively [57]. The energies of the states $5 S_{1 / 2}$ and $6 S_{1 / 2}$ are 0 and $20132.510 \mathrm{~cm}^{-1}$, respectively. The energies of the states $5 P_{1 / 2}$ and $5 P_{3 / 2}$ are 12578.950 and $12816.545 \mathrm{~cm}^{-1}$, respectively [58]. Using these values, we find $\left|\alpha_{J}^{(0)}\right| \cong 5.973 \times 10^{-38} \mathrm{~kg}^{-1} \mathrm{~s}^{4} \mathrm{~A}^{2}$.
[1] M. Göppert-Mayer, Über elementarakte mit zwei quantensprüngen, Ann. Phys. (Leipzig) 401, 273 (1931).

[2] W. Kaiser and C. G. B. Garrett, Two-Photon Excitation in $\mathrm{CaF}^{2}: \mathrm{Eu}^{2+}$, Phys. Rev. Lett. 7, 229 (1961).

[3] I. D. Abella, Optical Double-Photon Absorption in Cesium Vapor, Phys. Rev. Lett. 9, 453 (1962).

[4] D. M. Friedrich, Two-photon molecular spectroscopy, J. Chem. Educ. 59, 472 (1982).

[5] F. Schlawin, K. E. Dorfman, and S. Mukamel, Entangled twophoton absorption spectroscopy, Accounts Chem. Res. 51, 2207 (2018).

[6] W. Denk, J. Strickler, and W. Webb, Two-photon laser scanning fluorescence microscopy, Science 248, 73 (1990).

[7] L. Hilico, R. Felder, D. Touahri, O. Acef, A. Clairon, and F. Biraben, Metrological features of the rubidium two-photon standards of the BNM-LPTF and Kastler Brossel Laboratories, Eur. Phys. J. Appl. Phys. 4, 219 (1998).
[8] L. S. Vasilenko, V. P. Chebotaev, and A. V. Shishaev, Line shape of two-photon absorption in a standing-wave field in a gas, JETP Lett. 12, 113 (1970).

[9] B. Cagnac, G. Grynberg, and F. Biraben, Spectroscopie d'absorption multiphotonique sans effet Doppler, J. Phys. (Paris) 34, 845 (1973).

[10] F. Biraben, B. Cagnac, and G. Grynberg, Experimental Evidence of Two-Photon Transition without Doppler Broadening, Phys. Rev. Lett. 32, 643 (1974).

[11] M. D. Levenson and N. Bloembergen, Observation of TwoPhoton Absorption without Doppler Broadening on the $3 S-5 S$ Transition in Sodium Vapor, Phys. Rev. Lett. 32, 645 (1974).

[12] T. Nieddu, T. Ray, K. S. Rajasree, R. Roy, and S. Nic Chormaic, Simple, narrow, and robust atomic frequency reference at $993 \mathrm{~nm}$ exploiting the rubidium (Rb) $5 S_{1 / 2} F=2$ to $6 S_{1 / 2} F=2$ transition using one-color two-photon excitation, Opt. Express 27, 6528 (2019). 
[13] S. Wang, J. Yuan, L. Wang, L. Xiao, and S. Jia, A stable frequency standard based on the one-color two-photon $5 S-7 S$ transition of rubidium at $760 \mathrm{~nm}$, Laser Phys. Lett. 16, 125204 (2019).

[14] M. M Salour, Ultra-high-resolution two-photon spectroscopy in atomic and molecular vapors, Ann. Phys. (NY) 111, 364 (1978).

[15] S. Quabis, R. Dorn, M. Eberler, O. Glöckl, and G. Leuchs, Focusing light to a tighter spot, Opt. Commun. 179, 1 (2000).

[16] S. J. van Enk and H. J. Kimble, Single atom in free space as a quantum aperture, Phys. Rev. A 61, 051802(R) (2000).

[17] R. Mitsch, C. Sayrin, B. Albrecht, P. Schneeweiss, and A. Rauschenbeutel, Quantum state-controlled directional spontaneous emission of photons into a nanophotonic waveguide, Nat. Commun. 5, 5713 (2014).

[18] S. K. Ruddell, K. E. Webb, I. Herrera, A. S. Parkins, and M. D. Hoogerland, Collective strong coupling of cold atoms to an allfiber ring cavity, Optica 4, 576 (2017).

[19] F. Le Kien, S. S. S. Hejazi, T. Busch, V. G. Truong, and S. Nic Chormaic, Channeling of spontaneous emission from an atom into the fundamental and higher-order modes of a vacuum-clad ultrathin optical fiber, Phys. Rev. A 96, 043859 (2017).

[20] J.-B. Béguin, J. H. Müller, J. Appel, and E. S. Polzik, Observation of Quantum Spin Noise in a 1D Light-Atoms Quantum Interface, Phys. Rev. X 8, 031010 (2018).

[21] K. P. Nayak, J. Wang, and J. Keloth, Real-Time Observation of Single Atoms Trapped and Interfaced to a Nanofiber Cavity, Phys. Rev. Lett. 123, 213602 (2019).

[22] D. H. White, S. Kato, N. Német, S. Parkins, and T. Aoki, Cavity Dark Mode of Distant Coupled Atom-Cavity Systems, Phys. Rev. Lett. 122, 253603 (2019).

[23] N. V. Corzo, A. Raskop, J. Chandra, A. S. Sheremet, B. Gouraud, and J. Laurat, Waveguide-coupled single collective excitation of atomic arrays, Nature (London) 566, 359 (2019)

[24] R. Jones, G. Buonaiuto, B. Lang, I. Lesanovsky, and B. Olmos, Collectively Enhanced Chiral Photon Emission from an Atomic Array Near a Nanofiber, Phys. Rev. Lett. 124, 093601 (2020).

[25] S. M. Hendrickson, M. M. Lai, T. B. Pittman, and J. D. Franson, Observation of Two-Photon Absorption at Low Power Levels using Tapered Optical Fibers in Rubidium Vapor, Phys. Rev. Lett. 105, 173602 (2010).

[26] D. E. Jones, J. D. Franson, and T. B. Pittman, Ladder-type electromagnetically induced transparency using nanofiber-guided light in a warm atomic vapor, Phys. Rev. A 92, 043806 (2015).

[27] R. Kumar, V. Gokhroo, K. Deasy, and S. Nic Chormaic, AutlerTownes splitting via frequency up-conversion at ultralow-power levels in cold ${ }^{87} \mathrm{Rb}$ atoms using an optical nanofiber, Phys. Rev. A 91, 053842 (2015).

[28] R. Kumar, V. Gokhroo, and S. Nic Chormaic, Multi-level cascaded electromagnetically induced transparency in cold atoms using an optical nanofibre interface, New J. Phys. 17, 123012 (2015).

[29] T. Ray, R. K. Gupta, V. Gokhroo, J. L. Everett, T. Nieddu, K. S. Rajasree, and S. Nic Chormaic, Observation of the ${ }^{87} \mathrm{Rb} 5 S_{1 / 2}$ to $4 D_{3 / 2}$ electric quadrupole transition at $516.6 \mathrm{~nm}$ mediated via an optical nanofibre, New J. Phys. 22, 062001 (2020).

[30] F. Lei, G. Tkachenko, J. M. Ward, and S. Nic Chormaic, Complete Polarization Control for a Nanofiber Waveguide Using Directional Coupling, Phys. Rev. Appl. 11, 064041 (2019).

[31] M. Joos, A. Bramati, and Q. Glorieux, Complete polarization control for a nanofiber waveguide using the scattering properties, Opt. Express 27, 18818 (2019).

[32] G. Tkachenko, F. Lei, and S. Nic Chormaic, Polarisation control for optical nanofibres by imaging through a single lens, J. Opt. 21, 125604 (2019).

[33] R. Loudon, The Quantum Theory of Light (Oxford University Press, Oxford, 2000).

[34] D. A. Varshalovich, A. N. Moskalev, and V. K. Khersonskii, Quantum Theory of Angular Momentum (World Scientific, Singapore, 2008).

[35] A. R. Edmonds, Angular Momentum in Quantum Mechanics (Princeton University Press, Princeton, 1974).

[36] G. Grynberg and B. Cagnac, Doppler-free multiphotonic spectroscopy, Rep. Prog. Phys. 40, 791 (1977).

[37] K. D. Bonin and T. J. McIlrath, Two-photon electric-dipole selection rules, J. Opt. Soc. Am. B 1, 52 (1984).

[38] N. Melikechi and L. Allen, Two-photon electric-dipole selection rules and nondegenerate real intermediate states, J. Opt. Soc. Am. B 3, 41 (1986).

[39] K. Y. Bliokh, Y. Gorodetski, V. Kleiner, and E. Hasman, Coriolis Effect in Optics: Unified Geometric Phase and Spin-Hall Effect, Phys. Rev. Lett. 101, 030404 (2008).

[40] G. Tkachenko, I. Toftul, C. Esporlas, A. Maimaiti, F. Le Kien, V. G. Truong, and S. Nic Chormaic, Light-induced rotation of dielectric microparticles around an optical nanofiber, Optica 7, 59 (2020).

[41] F. Le Kien and A. Rauschenbeutel, Negative azimuthal force of nanofiber-guided light on a particle, Phys. Rev. A 88, 063845 (2013).

[42] F. Le Kien, J. Q. Liang, K. Hakuta, and V. I. Balykin, Field intensity distributions and polarization orientations in a vacuumclad subwavelength-diameter optical fiber, Opt. Commun. 242, 445 (2004).

[43] L. Tong, J. Lou, and E. Mazur, Single-mode guiding properties of subwavelength-diameter silica and silicon wire waveguides, Opt. Express 12, 1025 (2004).

[44] F. Le Kien, T. Busch, V. G. Truong, and S. Nic Chormaic, Higher-order modes of vacuum-clad ultrathin optical fibers, Phys. Rev. A 96, 023835 (2017).

[45] A. W. Snyder and J. D. Love, Optical Waveguide Theory (Chapman and Hall, New York, 1983).

[46] F. Biraben, M. Bassini, and B. Cagnac, Line-shapes in Dopplerfree two-photon spectroscopy. The effect of finite transit time, J. Phys. (Paris) 40, 445 (1979).

[47] J. M. Ward, A. Maimaiti, V. H. Le, and S. Nic Chormaic, Contributed review: Optical micro- and nanofiber pulling rig, Rev. Sci. Instrum. 85, 111501 (2014).

[48] K. S. Rajasree, T. Ray, K. Karlsson, J. L. Everett, and S. Nic Chormaic, Generation of cold Rydberg atoms at submicron distances from an optical nanofiber, Phys. Rev. Res. 2, 012038(R) (2020).

[49] S. M. Spillane, G. S. Pati, K. Salit, M. Hall, P. Kumar, R. G. Beausoleil, and M. S. Shahriar, Observation of Nonlinear 
Optical Interactions of Ultralow Levels of Light in a Tapered Optical Nanofiber Embedded in a Hot Rubidium Vapor, Phys. Rev. Lett. 100, 233602 (2008).

[50] H. J. Metcalf and P. van der Straten, Laser Cooling and Trapping (Springer, Berlin, 1999).

[51] F. Le Kien, V. I. Balykin, and K. Hakuta, Angular momentum of light in an optical nanofiber, Phys. Rev. A 73, 053823 (2006).

[52] E. Vetsch, D. Reitz, G. Sagué, R. Schmidt, S. T. Dawkins, and A. Rauschenbeutel, Optical Interface Created by Laser-Cooled Atoms Trapped in the Evanescent Field Surrounding an Optical Nanofiber, Phys. Rev. Lett. 104, 203603 (2010).

[53] M. Babiker, C. R. Bennett, D. L. Andrews, and L. C. Dávila Romero, Orbital Angular Momentum Exchange in the Interaction of Twisted Light with Molecules, Phys. Rev. Lett. 89, 143601 (2002).

[54] M. F. Andersen, C. Ryu, P. Cladé, V. Natarajan, A. Vaziri, K. Helmerson, and W. D. Phillips, Quantized Rotation of Atoms from Photons with Orbital Angular Momentum, Phys. Rev. Lett. 97, 170406 (2006).

[55] C. S. Wood, S. C. Bennett, D. Cho, B. P. Masterson, J. L. Roberts, C. E. Tanner, and C. E. Wieman, Measurement of parity nonconservation and an anapole moment in cesium, Science 275, 1759 (1997).

[56] B. J. Wundt, C. T. Munger, and U. D. Jentschura, Quantum Dynamics in Atomic-Fountain Experiments for Measuring the Electric Dipole Moment of the Electron with Improved Sensitivity, Phys. Rev. X 2, 041009 (2012).

[57] M. S. Safronova and U. I. Safronova, Critically evaluated theoretical energies, lifetimes, hyperfine constants, and multipole polarizabilities in ${ }^{87} \mathrm{Rb}$, Phys. Rev. A 83, 052508 (2011).

[58] A. Kramida, Y. Ralchenko, J. Reader, and NIST ASD Team, NIST Atomic Spectra Database, version 5.7.1 (National Institute of Standards and Technology, Gaithersburg, 2019), available at https://physics.nist.gov/asd. 